\title{
As reacções de Wassermann e de Sachs-Georgi na Lepra pelo
}

\author{
Dr. Antonio Eugenio de Arèa Leão.
}

(Do Instituto Oswaldo Cruz)

A reacção de WASSERMANN no sôro sanguineo dos leprosos, tem sido assumpto de pesquisas desde 1906, e a maioria dos auctores acredita ser ella posiliva em grande porcentagem no sôro daquelles doentes, mormente na fórma tuberculosa da molestia.

EITNER (1906), WECHSELMANN e MEYER (1908), LEVADITI E YAMANOU. CHI (1908), foram os primeiros a verificar a reacção de WASSERMANN positiva no sôro sanguineo dos leprosos.

JEANSELME (Presse Medicale, 1912, p. 269) examinando o sôro de leprosos e syphiliticos com as reacções de EITNER e WASSERMANN chegou ao seguinte resultado: em 40 sôros de syphiliticos em plena actividade da molestia, 40 vezes a reacção de WASSERMANN foi positiva e 33 vezes deu positiva a reacção de EITNER. Assim obteve 85,5 \% de reacções positivas no sôro de syphiliticos, usando o antigeno de EITNER.

No homem são, ou no syphilitico em latencia, as duas reacções, de WAS-
SERMANN e de EITNER, foram negativas em $100 \%$ dos casos.

A reacção de EITNER quando positiva nos leprosos se acompanha muitas vezes de WASSERMANN posilivo. Assim, de seis sôros leprosos examinados por JEANSELME, tres apresentaram as duas reacções, de EITNER e WASSERMANN, positivas e os outros tres negativas; um outro examinado no periodo apyretico da lepra e no decurso de uma "poussée» maculosa, deu uma reacção de EITNER positiva e reacção de WASSERMANN negativa. Conclue, então, JEANSELME, que os dois antigenos, de EITNER e de WASSERMANN, podem um e outro desviar o complemento, tanto com o sôro syphilitico como com o sôro leproso.

Mas a sensibilidade da reacção é maior na lepra com o antigeno de EITNER e na syphilis com o antigeno de WASSERMANN. Disso resulta que as substancias existentes nos sôros, lepro. so e syphilitico, sobre as quaes agem estes dois antigenos, têm propriedades biologicas visinhas, mas não identicas. 
Quando os sôros syphiliticos contêm muitas reaginas syphiliticas, qualquer antigeno, mesmo de fraco poder fixador, desvia o complemento; agora nos sôros com poucas reaginas syphiliticas só os antigenos muito sensiveis as podem descobrir. Este facto se verifica quando se pratica a reacção com antigenos leprosos e syphiliticos de valores differentes.

Os sôros leprosos muito sensiveis o são tambem com os antigenos syphiliticos mediocres.

Um facto interessante assignalado por JEANSELME é que a reacção de WASSERMANN desapparece no sôro dos syphiliticos submettidos ao tratamento especifico, emquanto o sôro leproso em nada se altera com o tratamento antisyphilitico. Isso constitue, não resta duvida, um meio de afastar a syphilis nos casos examinados; mas nem sempre o desapparecimento da reacção no sôro sanguineo dos syphiliticos se dá com rapidez, embora seja empregada a therapeutica anti-syphilitica mais energica. A reacção por vezes se conserva positiva, em nada diminuindo de intensidade, independente do tratamento.

PHOTINUS e MICHAELIDES (Lepra, v. XII, f. 4, 1912, pp. 217-219), praticaram a reacção de WASSERMANN em 204 sôros leprosos; destes, 115 reagiram positivamente e 89 foram negalivas. A maior porcentagem de reacções positivas foi observada na fórma tuberculosa da lepra $(75,9 \%)$, com $21,4 \%$ de reacçoes negativas; na fórma nervosa $35 \%$ de positivas e $62 \%$ de reacções negativas; na fórma mixta $75 \%$ de reacções positivas e $25 \%$ de reacções negativas.

Como antigeno foi usado o figado de feto heredo-syphilitico e o extracto alcoolico de lepromas.

MATHIS e BONJEAN (Bull. Soc. Path. exot., 1915, p. 225), examinaram o sôro de leprosos do TONKIN, usando a technica do Instituto Pasteur de Lille, que é a technica de CALMETTE e MAS-
SOL (doses crescentes de alexina e quantidade fixa de antigeno).

Como antigeno usaram extracto alcoolico de varios figados de feto heredosyphiliticos, diluido em agua physiologica, de maneira que 0,5 c.c. da diluição continha 5 unidades de antigeno.

Nos 41 sôros leprosos examinados obtiveram 40 reacçães nitidamente negativas; o unico caso restante, onde a reacção foi positiva, tratava-se de um leproso syphilitico. Verificaram as propriedades anticomplementares do sôro leproso, alguns absorvendo por si só 4 a 5 unidades de alexina.

Chegam á conclusão que a reacção de WASSERMANN é constantemente negativa na lepra.

SLATINEANU E DANIELOPOLU (C. R. S. Biol., v. 1, 332), experimentaram a fixação do complemento no sôro dos leprosos, usando como antigeno a lecithina de ovo da casa Kahlbaum de Berlim, emulsionada em agua physiologica na proporção de $1 \%$ e addicionada de acido phenico. Com esse antigeno examinaram o sôro de 21 leprosos, dos quaes 10 fixaram completamente a alexina; em dois casos a reacção de fixação foi fraca e as 9 restantes foram negativas. No liquor de 20 leprosos examinados não encontraram a minima fixação.

Observaram mais, que os resultados não concordam, quando se usam como antigenos a lecithina e um antigeno syphilitico; havendo sôros que fixam o complemento quando usada a lecithina e deixam de fixal-o em presença de um antigeno syphilitico e vice-versa.

Chegam á conclusão de que é grande a porcentagem de reacçóes de WASSERMANN positivas no sôro de doentes de lepra, usando a lecithina como antigeno, a fixação no liquor sendo nulla com esse mesmo antigeno.

RECCIO (Sanidad y Beneficiencia, 1909 , p. 277) examinaram 20 leprosos 14 com manifestações cutanẹas, 4 
de lepra trophoneurotica e dois diagnosticados de syringomyelia; $\left(^{*}\right)$ dos quatro casos de lepra trophoneurotica, um deu reacção fracamente positiva, outro duvidosa e os restantes negativas. $\mathrm{Na}$ syringomyelia a reacção foi negativa.

PASINI (L'Ospedale Maggiore, t.IV, 1909) examinou o sôro, o liquior e a urina de 3 leprosos com varios antigenos (extracto de nodulo leproso, figado e baço he:eđo-syphiliticos e suspensão de lecithina). Conclue que a reacção de WASSERMANN na lepra não é uma reacção biologica especifica, mas uma reacção physicochimica de natureza ainda indeterminada, pois é positiva tanto com os antigenos usados para a syphilis como com o extracto de lepromas. A urina dá quasi sempre resultados positivos, que são sem valor, porque nos individuos normaes tambem dá resultados positivos em $80 \%$ dos casos.

EHLER e BOURRET ((Bull. Soc. Path. exot., t. 11, 1909, p. 520), pratica. ram a reacção de WASSERMANN no sôro de 47 leprosos (9 de lepra tuberculosa, 6 de lepra mixta, 29 de lepra nervosa e 3 duvidosos) empregando como antigeno o coração de cobaya normal.

Obtiveram na lepra tuberculosa uma reacção positiva completa e oito positivas parciaes em graus diversos; na lepra mixta 6 reacções positivas parciaes; na lepra nervosa 2 reacçóes positivas completas, 25 parcialmente positivas e 2 negativas.

Não encontrou explicação para a differença observada no grau da reacção, nem na fórma, na antiguidade ou no estado de evolução da molestia. Praticando a reacção novamente um mez após a primeira sangria em 14 dos seus doentes, cinco vezes a fixação obtida não coincidiu com a primeira reacção.

(') Só encontraram uma reacção negativa nos 14 casos de manifestações cutaneas da molestia, sendo positivas todas as restantes.
FRUGONE e PISANI (Berl. Klin. Woch., 1909, p. 1530-1531), examinaram 11 sôros leprosos com differentes antigenos (figado de feto syphilitico, nodulos leprosos contendo bacillos, sarcoma, carcinoma, tuberculina, emulsão de B. de KOCH, sôro antituberculoso de HOECHST). Em mais da metade dos casos encontraram fixação do complemento com o extracto de nodulos leprosos; não houve pelo contrario nenhuma fixação com este antigeno e o sôro normal ou proveniente de outra molestia. Obtiveram reacções de WASSERMANN posilivas em alguns dos sôros examinados sem que os doentes tivessem o menor symptoma de syphilis.

Resultados positivos tambem foram observados com outros antigenos, como a tuberculina, o B. de KOCH ou o extracto de tumores e isso nos leprosos que não eram nem tuberculosos, nem portadores de neoplasma.

ELIAS BERG (Deutsch. mediz. Woch., 1909., p. 1922-1923), em 31 doentes de lepra tubercculosa, obteve a reacção de WASSERMANN positiva 25 vezes $(80,6$ $\%$ ); em 190 casos de lepra nervosa, só tres deram reacções positivas $(15,8 \%)$. Notou ainda o poder impediente do sôro leproso na đóse de 0,4 ; facto não observado geralmente para os outros sôros.

AKERBERG, ALMKWIST e JUNDELL (Lepra, t. IX, f. 3, 1910), affirmam que o sôro leproso só desvia o complemento em presença de um antigeno syphilitico em $15 \%$ dos casos.

BABES (II Conference de la lepre, t. 111, p. 131), acha que a lepra tuberculosa, dá melhor que a fórma nervosa da molestia, reacções de WASSERMANN positivas, usando-se como antigeno o figado de feto heredo-syphilitico ou o coração de cobaya.

MEYER (II Conference de la lepre, t. 111, p. 334), affirma que a reacção de WASSERMANN é positiva em $70 \%$ dos casos de lepra tuberculosa. A fixação do 
complemento se dá ainda muito mais vezes na lepra tuberculosa, quando se usa a tuberculina como antigeno.

O diagnostico differencial entre a lepra e a syphilis deve ser feito com a tuberculina.

HANN e GRIJNS (Geneesk. Tijdschr. Med. Indie, t. L, f. 4, 1910), de 18 casos de lepra examinados (anesthesica, tu. berculosa e maculo-tuberculosa), sete desviaram o complemento, nove reacções negativas e seis duvidosas.

BABES (Zeitschr. f. Imm. I. Orig., t. VII, 1910 , p. 578-623), affirma que a maior parte dos sôros leprosos fixa o complemento com um antigeno syphilitico, mormente quando se trata da fórma tuberculosa da molestia; na fórma anesthesica a reacção positiva se observa muito raramente. Pelo contrario o sôro syphilitico não fixa o complemento, senão muito raramente, em presença de um antigeno leproso.

JOLTRAIN (Nouvelles Methodes de Sero-Diagnostic, p. 245), obteve 14 vezes a reacção de WASSERMANN positiva em 22 leprosos que examinou. Assim se exprime JOLTRAIN sobre os sôros leprosos: "Les divergences qui existent entre ces différents auteurs nous paraissent surtout provenir de ce fait que le sérum des lépreux a certainement une composition trés spéciale et sa richesse en certaines substances, protéines ou globulines, fait que, très souvent, il est fixant de lui-même; nous l'avons, pour notre part, trouvé avec ces propriétés dans une proportions de 26 p. 100.

"Un autre caractére important du sérum des lépreux, c'est qu'il est polyfixant, et il semble, à ce point de vue, que tous 'les auteurs soient d'accord. Il donne, en effet, la réaction de BORDET et GENGOU avec un très grand nombre d'antigènes différents. Nous l'avons vu dévier le complément en employant comme antigènes l'extrait alcoo'ique de foie syphilitique un extrait de carcinome, d'épithelioma, de mycosis fongoide, de tuberculome, enfin avec certains lécithines, la luberculine, le Sporotrichum beurmanni, l'actinomyces et certains microbes comme le pneumocoque et le bacille d'EBERTH».

A maioria dos autores concorda, pois, que a reacção de WASSERMANN é posiliva na lepra em uma porcentagem bem grande.

As propriedades poly-fixantes do sôro leproso, que o fazem desviar 0 complemento em presença dos antigenos os mais diversos, alliadas ao seu poder impediente natural, explicam provavelmente a grande percentagem de reacções de WASSERMANN positivas na lepra. Isso, entretanto, não tira de modo nenhum o valor da reacção de WASSERMANN na syphilis. Não sendo embora especifica, antigenos e anticorpos verdadeiros, mas reacções que se passam entre lipoides do antigeno e os existentes no sôro, o certo é que na syphilis ella é de uma frequencia extraordinaria.

$\mathrm{Na}$ forma tuberculosa da lepra que dá, em maior percentagem, reacção de WASSERMANN posiliva, o diagnostico differencial com a syphilis não offerece difficuldade e o exame microscopico dos nodulos mostrará certamente a presença do B. de HANSEN, sendo este muito constante nas lesões cutaneas desta molestia e a sua pesquisa de facil execução.

A prova therapeulica de JEANSELME para afastar a syphilis, tratando os pacientes com os medicamentos antisyphililicos e observando nestes o desapparecimento da reacção de WASSERMANN e a sua permanencia nos casos de lepra, não pode merecer confiança absoluta. Ha casos de syphilis pura e WASSERMANN-resistentes, mantendo-se a reacção com o mesmo grau de fixação independente do tratamento o mais ener- 
gico. Infelizmente estes casos não são raros.

Assim a prova therapeutica poderá ser lentada, não se devendo, entretanto, della esperar tudo.

O desvio do complemento com o extracto de leproma, não é tambem uma prova segura de diagnostico differencial das duas molestias, porque este antigeno desvia ás vezes o complemento com o sôro syphilitico.

Assim só a clinica poderá resolver a questão, auxiliada nos casos possiveis da 'pesquisa directa do bacillo nas lesões.

Tivemos occasião de praticar as reaccões de WASSERMANN e SACHSGEORGI no sôro sanguineo de 50 leprosos internados do Hospital dos Lazaros do Rio de Janeiro, a cargo do Professor Dr. FERNANDO TERRA, que nos permitliu gentilmente taes pesquisas e a quem somos muito grato por tudo. Destes 50 doentes, 8 eram de lepra maculosa; 233 de lepra tuberculosa; 17 de lepra nervosa; 1 de lepra mixta e 1 de lepra maculo-anesthesica.

Na reacção de WASSERMANN usamos os seguintes antigenos: figado de feto heredo-syphilitico, coração humano cholesterinado, coração de boi cholesterinado, lipoides insoluveis na acetona, segundo o processo de NOGUCHI, lipoides insoluveis na acetona, segundo a technica de BORDET e o extracto ethereo de LESSER.

Todos estes antigenos experimentados largamente com sôros syphiliticos sempre se mostraram muito sensiveis.

O poder impediente de todos elles era previamente dosado.

Os sôros leprosos eram examinados, no mesmo dia da sangria, para evitar que augmentassem as suas qualidades impedientes, e quando o exame não podia ser feito no mesmo dia decantava- mos o sôro que era em seguida inactivado em banho-maria a $56 \circ \mathrm{C}$., durante 15 minutos. Feito isto os sôros eram guardados na geladeira e a reacção feita no dia seguinte.

O complemento usado na reacção era o sôro fresco de cobaya normal, retirado no mesmo dia da reacção para que nada perdesse do seu poder. Para termos "um complemento mais homogeneo sangravamos 10 a 20 cobayas.

O sôro hemolytico usado foi o de coelho para os globulos vermelhos de carneiro; sôro este de grande poder hemolytico, hemolysando em meia hora 1 c.c. de globulos de carneiro a $5 \%$, na diluição de 1 para 6000 . Utilizavamos sempre na reacção 3 a 4 dóses hemolyticas, que era o sufficiente para vencer o poder impediente natural do sôro leproso.

Os globulos de carneiro lavados varias vezes na agua physiologica eram emulsionados nesta na proporção de $5 \%$.

A technica usada foi a seguinte: sete tubos para cada reacção, cada um delles recebia 0,2 de sôro leproso, em seguida 0,2 de cada antigeno, a excepção do ultimo que ficava como testemunho do sôro não recebendo antigeno. $\left(^{*}\right)$ Completavamos o volume de 1 c.c. com agua physiologica, em todos os tubos e juntavamos emfim 1 c.c. de complemento (sôro de cobaya diluido, em agua physiologica, a 1 para 10).

A quantidade de 1 c.c. de complemento usada na reacção, era deste modo em excesso e sufficiente para vencer o poder impediente natural do sôro leproso.

Os tubos assim preparados, representando, cada um, um antigeno diffe-

(*) Esta dose de 0,2 dos diversos antigenos empregados, representava quatro a cinco unidades anti genicas e para obtel-a os antigenos eram dosados préviamente com sôros de individuos syphiliticos e empregados em diluição tal que o volume de 0,2 contivesse as unidades antigenicas referidas, 
rente, eram levados ao banho-maria á $37 \circ \mathrm{C}$. e ahi soffriam a primeira incubabação durante 1 hora. No fim deste lempo juntavamos os globulos de carneiro sensibilizados com 3 a 4 unidades hemolysantes.

A leitura da reacção era feita no fim de uma hora, quando todos os tubos testemunhos se achavam completamente hemolysados.

A hemolyse se passa vagarosamente quando se junta o systema hemolytico; o sôro leproso mesmo recente, isto é, colhido e examinado no mesmo dia tem qualidades impedientes bem accentuadas, e foi este o motivo que nos levou a prolongar o lempo para a leitura final da reacção.

Comparavamos então o grau de fixação com os 6 antigenos usados e os resultados damos em um quadro áparte, designando pelo maior ou menor numero de cruzes a maior ou menor fixação, e finalmente um traço horizontal onde aquella era nulla. Nelle se observam com facilidade como se comportam os differentes antigenos usados em presença do sôro leproso. Assim é que os antigenos que dão maior fixação com o sôro leproso são: o figado de feto heredo-syphilitico, coração humano e coração de boi cholesterinados. Os antigenos de lipoides insoluveis na acetona, preparados, quer pelo processo de NOGUCHI ou o de BORDET, fixam menos, e o antigeno de LESSER é de todos o que dá menor fixação.

Estes antigenos experimentados com sôros syphiliticos todos se comportavam quasi identicamente; mas aqui, como se vê no quađro, ha uma certa discordancia entre elles no grau de fixação.

A percentagem maior de reacções de WASSERMANN positivas é encontrada nas fórmas tuberculosas e maculosas da molestia, mormente na ultima. Todos os doentes por nós observados, não tinham na sua historia clinica a syphilis e a maioria delles, tratados com 914 não apresentavam melhora e nada influia a ađministracção do medicamento nos resultados da reacção.

Na lepra nervosa a percentagem de reacções de WASSERMANN positivas é muito menor e isso tem sido verificado por todos os auctores.

A reacção de precipilação de SACHSGEORGI, ultimamente aconselhada por estes auctores para o diagnostico da syphilis, é nesta molestia đe uma concordancia muito grande com a reacção de WASSERMANN, como verificámos em trabalho anterior; estudando comparativamente 2000 casos, procurámos verificar se a reacção de SACHS-GEORGI era tambem, como a reacção de WASSERMANN, positiva em alta dose no sôro leproso.

Seguimos, praticando esta reacção, a mesma technica dos auctores, usando como antigeno o coração de boi extrahido com alcool na proporção de 1 para 5 . Este extracto nos deu os melhores resultados com os sôros syphiliticos.

O sôro leproso era diluido em agua physiologica a 8,5 p. 1000 na proporção de 1 para 10 . A 1 c.c. do sôro assim diluido juntavamos 0,5 do antigeno, tambem diluido em agua physiologica a 1 para 6.

Os tubos permaneciam na estufa á 37 oC., durante 24 horas, quando então os resultados eram lidos.

Os resultados são mais ou menos identicos aos da reacção de WASSERMANN, havendo entretanto um maior numero de reacções negativas com a reacção de SACHS-GEORGI.

Com a reacção de WASSERMANN, o maior numero de reacções posilivas é dado pelas fórmas tuberculosa e maculosa da lepra, a fórma nervosa desviando o compiemento em muito menor numero. 
1-F., sexo masculino, 40 annos de molestia. Lipra nervosa. Reacção de WASSERMANN : positiva. Reacção de SACHSGEORGI: negativa.

2-J. de M., 39 annos, 20 annos de molestia. Lepra nervosa. Reacção de WASSERMANN : negativa. Reacção de SACHS-GEORGI : negativa.

3-A., 42 annos, 7 annos de molestia. Lepra nervosa. Reacção de WASSERMANN: negativa. Reacção de SACHSGEORGI: negativa.

4-M., 24 annos, 5 annos de molestia. Lepra tuberculosa. Reacção de WASSERMANN : positiva. Reacção de SACHSGEORGI : negativa.

5-A., 38 annos, 6 annos de molestia. Lepra maculosa. Reacção de WASSEMANN: positiva. Reacção de SACHSGEORGI : positiva.

6-J. V., 27 annos, 6 annos de molestia. Lepra maculosa. R. de W. : positiva. R. S. G. : positiva.

7-R. P., 33 annos, 2 annos de molestia. Lepra maculosa. R de W. : positiva. R. de S. G. : positiva.

8-V. P., 30 annos, 1 anno de molestia. Lepra nervosa. R.de W. : positiva. R. de S. G. : positiva.

9-N. V., 30 annos, 7 annos de molestia. Lepra nervosa. R. de W. : negativa. R. de S. G.: negativa.

10 -J. N., 28 annos, 10 annos de molestia. Lepra tuberculosa. R. de W.: posiliva. R. de S. G: negativa.

11-A. L., 20 annos, 3 annos de molestia. Lepra nervosa. R. de W. : negativa. R. de S. G. : negativa.

12-C. S., 15 annos, 3 annos de molestia. Lepra tuberculosa. R. de W. : negativa. R. de S. G. : negativa.

13-J. P., 34 annos, 17 annos de mo. lestia. Lepra tuberculosa. R. de W. : positiva. R. de S. G. : positiva.

14-B. L., 29 annos, 10 annos de molestia. Lepra tuberculosa. R. de W. : positiva, $R$, de $S_{\text {. }} G_{\text {s }}$ : posiliva.
15-M. L., 25 annos, 5 annos de moIestia. Lepra maculosa. R. de W. : positiva. R. de S. G. : positiva.

16-E. N., 50 annos, 5 annos de molestia. Lepra tuberculosa. R. de W. : positiva. R. de S. G. : negativa.

17-A. D., 15 annos, 1 anno de molestia. Lepra tuberculosa. R. de W. : negativa. R. de S. G. : negativa.

18-J. P., 28 annos, 16 annos de molestia. Lepra tuberculosa. R. de W. : negativa. R. de S. G. : negativa.

19-M. G., 65 annos, 12 annos de molestia. Lepra tuberculosa. R. de W. : negativa. R. de S. G. : negativa.

20-J. M., 58 annos, 6 annos de molestia. Lepra tuberculosa. R. de W. : positiva. R. de S. G. : positiva.

21-J. C., 45 annos, 7 annos de molestia. Lepra tuberculosa. R. de W. : positiva. R. de S. G. : positiva.

22-Z., 39 annos, 12 annos de molestia. Lepra maculo-anesthesica. R. de W. : positiva. R. de S. G. : negativa.

23-L. J., 43 annos, 11 annos de molestia. Lepra nervosa. R. de W. : negativa. R. de S. G. : negativa.

24 -J. R., 32 annos, 6 annos de molestia. Lepra tuberculosa. R. de W. : positiva. R. de S. G. : positiva.

25-S. de A., 25 annos, 5 annos de molestia. Lepra tuberculosa. R. de W. : positiva. R. de S. G. : positiva

26-L. de S., 34 annos, 14 annos de molestia. Lepra nervosa. R. de W. : negativa. R. de S. G. : negativa.

27-J. de S., 49 annos, 20 annos de molestia. Lepra tuberculosa. R. de W. : positiva. R. de S. G.: positiva.

28-B. M., 15 annos, 2 annos de molestia. Lepra maculosa. R. de W. : positiva. R. de S. G. : negativa.

29-J. S., 30 annos, 7 annos de molestia. Lepra nervosa. R. de W. : negativa. R. de S. G. : negativa.

30 -V. G., 62 annos, 12 annos de molestia. Lepra nervosa. R. de .W. : negativa. R, de S. G. : negativa. 
31-R. A., 38 annos, 2 annos de moIeslia. Lepra tuberculosa. R. le W. : po. siliva. R. de S. G. : negativa.

32-P. P., 59 annos, 4 annos de molestia. Lepra nervosa. R. de W. : negativa. R. de S. G. : negativa.

33-J. M., 18 annos, 6 annos de molestia. Lepra tuberculosa. R. de W. : negativa. R. de S. G. : negativa.

34-J. de A., 62 annos, 15 annos de morestia. Lepra nervosa. R. de W. : positiva. R. de S. G. : positiva.

35-C. S., 21 annos, 4 annos de molestia. Lepra nervosa. R. de W. : positiva. R. de S. G. : negativa.

36-G. G., 57 annos, 34 annos de molestia. Lepra nervosa. R. de W. : negativa. R. de S. G. : negativa.

37-R. L., 32 annos, 13 annos de molestia. Lepra tulerculosa. R. de W. : positiva. R. de S. G. : positiva.

$38-F . ~ R ., 39$ annos, 9 annos de mo lestia. Lepra Lu' e:culosa. R. de W. : posiliva. R. de S. G. : negativa.

39-J. R., 31 annos, 5 annos de molestia. Lepra maculosa. R. de W. : posisitiva. R. de S. G. : positiva.

40-J. B., 25 annos, 8 annos de molestia. Lepra tuberculosa. R. de W. : pova. R. de S. G. : positiva.
41-J. M., 40 annos, 13 annos de molestia. Lepra nervosa. R. de W. : negatitiva. R. de S. G. : positiva.

42-J. M., 16 annos, 9 annos de molestia. Lepra tucerculosa. R. de W. : ne. gativa. R. de S. G. : negativa.

43-J. M., 11 annos, 1 anno de molestia. Lepra maculosa. R. de W. : negativa. R. de S. G. : negativa.

44-G. B., 13 annos, 4 annos de molestia. Lepra tuberculosa. R. de W. : negativa. R. de S. G. : negativa.

45-J. M., 14 annos, 2 annos de molestia. Lepra tuijerculosa. R. de W. : positiva. R. de S. G. : negativa.

46.-M. F. G., 15 annos, 4 annos de molestia. Lepra maculosa. R. de W.: poosiliva. R. de S. G.: negativa.

47-A. C., 11 annos, 5 annos de molestia. Lepra tuberculosa. R. de W. : negativa. R. de S. G. : negativa.

48-M. F. R., 59 annos, 9 annos de molestia. Lepra mixta. R. de W. : negativa. R. de S. G. : negativa.

49-H. L., 35 annos, 12 annos de molestia. Lepra nervosa. R. de W. : negativa. R. de S. G. : negativa.

50-B. A., 32 annos, 4 annos de molestia. Lepra nervosa. R. de W. : negativa. R. de S. G. : negativa. 


\begin{tabular}{|c|c|c|c|c|c|c|}
\hline & $\begin{array}{l}\text { Figado de } \\
\text { feto }\end{array}$ & $\begin{array}{l}\text { Coração hu- } \\
\text { mano }\end{array}$ & $\begin{array}{l}\text { Coração de } \\
\text { boi }\end{array}$ & $\begin{array}{c}\text { Antigeno de } \\
\text { Noguchi }\end{array}$ & $\begin{array}{l}\text { Antigeno de } \\
\text { Bordet }\end{array}$ & $\begin{array}{l}\text { Antigeno de } \\
\text { Lesser }\end{array}$ \\
\hline 1 & + & ++ & $+t$ & + & + & - \\
\hline 2 & - & - & - & - & - & - \\
\hline 3 & - & - & - & - & - & - \\
\hline 4 & + & ++ & ++ & - & $+t$ & - \\
\hline 5 & $+t+$ & +++ & $+t+$ & - & $+t+$ & - \\
\hline 6 & $+t+t$ & $+t+t$ & $+t+t$ & + & $+t+t$ & + \\
\hline 7 & $+t+t$ & $+t+t$ & $+t+t$ & + & $+t+t$ & - \\
\hline 8 & $+t$ & $+t$ & $t+$ & + & $+t$ & + \\
\hline 9 & - & - & - & - & $\cdots$ & - \\
\hline 10 & - & $+t+$ & $+t+$ & - & $+t$ & - \\
\hline 11 & - & - & - & - & - & - \\
\hline 12 & - & - & - & - & 一 & - \\
\hline 13 & $+++t$ & $+t+t$ & $+t+t$ & $t+t+$ & $t-t+t$ & $+t+t$ \\
\hline 14 & $+t$ & $+t+$ & $+t+$ & - & $t+$ & - \\
\hline 15 & $+t+t$ & $+t+t$ & $+t+t$ & $+t+t$ & $+t+t$ & $+t+t$ \\
\hline 16 & + & $+t+$ & $t+t+$ & - & - & - \\
\hline 17 & - & - & - & - & - & - \\
\hline 18 & - & - & - & - & - & - \\
\hline 19 & - & - & - & - & - & - \\
\hline 20 & $+t+t$ & $+t+t$ & $+t+t$ & $+t+t$ & - & - \\
\hline 21 & $+t+t$ & $t+t+$ & $+t+t$ & $+t+t$ & - & - \\
\hline 22 & - & $+t$ & $+t+$ & - & - & - \\
\hline 23 & - & - & - & -- & - & - \\
\hline 24 & $+t+t$ & $+t+t$ & $+t+t$ & $++t+$ & $t+t+$ & $t+t+$ \\
\hline 25 & $+t+t$ & $+t+t$ & $+t+t$ & $t+t$ & $+t+t$ & $+t+t$ \\
\hline 26 & - & - & - & - & - & - \\
\hline 27 & $+t$ & $+t+t$ & $t+t$ & ++ & ++ & - \\
\hline 28 & $+t+t$ & $+t+t$ & $+t+t$ & - & - & - \\
\hline 29 & - & - & - & - & - & - \\
\hline 30 & - & - & - & - & - & - \\
\hline 31 & + & $+t+t$ & $t+t+$ & - & - & - \\
\hline 32 & - & - & - & - & - & - \\
\hline 33 & - & - & - & - & - & - \\
\hline 34 & $+t+$ & +++ & $t+t$ & +++ & $+t+$ & - \\
\hline 35 & $\ldots$ & ++ & $t+$ & - & - & - \\
\hline 36 & - & - & - & - & - & - \\
\hline 37 & $++t$ & $+t+$ & $+t+$ & $+t+$ & $++t$ & - \\
\hline 38 & - & $+t$ & ++ & - & - & - \\
\hline 39 & $++t$ & $+t+$ & +++ & $++t$ & $+t+$ & +++ \\
\hline 40 & +++ & $++t$ & +++ & $+t+$ & - & - \\
\hline 41 & - & - & - & - & - & - \\
\hline 42 & - & - & - & - & - & - \\
\hline 43 & - & - & - & - & - & - \\
\hline 44 & - & - & - & - & - & - \\
\hline 45 & + & $+t$ & $+t$ & $t+$ & - & - \\
\hline
\end{tabular}




\begin{tabular}{c|c|c|c|c|c|c}
\hline & $\begin{array}{c}\text { Figado de } \\
\text { feto }\end{array}$ & $\begin{array}{c}\text { Coração hu- } \\
\text { mano }\end{array}$ & $\begin{array}{c}\text { Coração de } \\
\text { boi }\end{array}$ & $\begin{array}{c}\text { Antigeno de } \\
\text { Noguchi }\end{array}$ & $\begin{array}{c}\text { Antigeno de } \\
\text { Bordet }\end{array}$ & $\begin{array}{c}\text { Antigeno de } \\
\text { Lesser }\end{array}$ \\
\hline \hline 46 & ++ & ++ & ++ & - & - & - \\
47 & - & - & - & - & - & - \\
48 & - & - & - & - & - & - \\
49 & - & - & - & - & - & - \\
50 & - & - & - & - & - & - \\
\hline
\end{tabular}

REACÇÃO DE SACHS-GEORGI. .

No. 1 Nesaliva.

* 2 Negativa.

- 3 Negativa.

- 4 Negativa.

" 5 Positiva.

* 6 Positiva.

* 7 Positiva.

" 8 Positiva.

- 9 Negativa.

" 10 Negativa.

* 11 Negativa.

- 12 Neggativa.

* 13 Positiva.

* 14 Positiva.

* 15 Positiva.

* 16 Negativa.

* 17 Negativa.

" 18 Negativa.

" 19 Negativa.

- 20 Positiva.

" 21 Positiva.

* 22 Negativa.

- 23 Negativa.

* 24 Positiva.
- 25 Positiva.

- 26 Negativa.

* 27 Positiva.

" 28 Negaliva.

- 29 Negativa.

" 30 Negativa.

" 31 Negativa.

- 32 Negativa.

* 33 Negativa.

" 34 Positiva.

- 35 Negativa.

- 36 Negativa.

" 37 Positiva.

- 38 Negativa.

* 39 Positiva.

* 40 Positiva.

* 41 Positiva.

* 42 Negativa.

- 43 Negativa.

* 44 Negativa.

- 45 Negativa.

- 46 Negativa.

* 47 Negativa.

- 48 Negativa.

- 49 Negativa.

" 50 Negativa. 


\section{CONCLUSOES.}

1.-A reacção de WASSERMANN na lepra é posiliva muitas vezes; $50 \%$ com os antigenos de figado de feto heredo-syphilitico, coração humano e coração de boi cholesterinados; $32 \%$ com os antigenos de lipoides insoluveis na acetona (processo de NOGUCHI ou de BORDET); e $14 \%$ com o antigeno de LESSER (extracto ethereo de orgão?

2.-O grau de fixação é muito variavel com os diversos antigenos usados. Entretanto, são os antigenos preparados com figado de feto heredo-syphilitico e coração de boi cholesterinado, os que dão maior fixação.

3.-O maior numero de reacções de WASSERMANN positivas é dado pelas fórmas maculosa $(87,5$
$\%$ ) e tuberculosa $(65,2 \%)$ da lepra. A fórma nervosa da molestia figura com uma percentagem de $17,7 \%$ de reacções positivas.

4.-A causa da fixação do complemento pelo sôro leproso em presença de um antigeno syphilitico, está certamente nas qualidades poly-fixantes daquelle sôro.

5.-A reacção de SACHS-GEORGI na lepra dá uma percentagem total de $36 \%$ de reacções positivas.

6.-A maior fercentagem de reacções de SACHS-GEORGI posilivas na lepra é dada pela fórma maculosa da molestia $(62,5 \%$ ), vindo depois a fórma tuberculosa $(39,2 \%)$. A fórma nervosa dá uma percentagem de $17,7 \%$ de reaç̧ões de SACHS-GEORGI positivas. 